Spectral Element Agglomerate Algebraic Multigrid Methods for Elliptic Problems with High-Contrast Coefficients

Y. Efendiev, J. Galvis, P.S. Vassilevski

November 12, 2009

19th International Conference on Domain Decomposition Methods

Zhangjiajie, China

August 17, 2009 through August 22, 2009 
This document was prepared as an account of work sponsored by an agency of the United States government. Neither the United States government nor Lawrence Livermore National Security, LLC, nor any of their employees makes any warranty, expressed or implied, or assumes any legal liability or responsibility for the accuracy, completeness, or usefulness of any information, apparatus, product, or process disclosed, or represents that its use would not infringe privately owned rights. Reference herein to any specific commercial product, process, or service by trade name, trademark, manufacturer, or otherwise does not necessarily constitute or imply its endorsement, recommendation, or favoring by the United States government or Lawrence Livermore National Security, LLC. The views and opinions of authors expressed herein do not necessarily state or reflect those of the United States government or Lawrence Livermore National Security, LLC, and shall not be used for advertising or product endorsement purposes. 


\title{
Spectral element agglomerate algebraic multigrid methods for elliptic problems with high-contrast coefficients
}

\author{
Yalchin Efendiev ${ }^{1}$, Juan Galvis ${ }^{1}$, and Panayot S. Vassilevski ${ }^{2}$ \\ ${ }^{1}$ Department of Mathematics, Texas A\&M University, College Station, TX \\ 77843-3368, $\mathrm{USA}^{\ddagger}$ \\ 2 Center for Applied Scientific Computing, Lawrence Livermore National \\ Laboratory, L-560, Livermore, CA 94550. §
}

\section{Summary}

We apply a recently proposed ([3]) robust overlapping Schwarz method with a certain spectral construction of the coarse space in the setting of element agglomeration algebraic multigrid methods (or agglomeration AMGe) for elliptic problems with high-contrast coefficients. Our goal is to design multilevel iterative methods that converge independent of the contrast in the coefficients. We present simplified bounds for the condition number of the preconditioned operators. These bounds imply convergence that is independent of the contrast. In the presented preliminary numerical tests, we use geometric agglomerates; however, the algorithm is general and offers some simplifications over the previously proposed spectral agglomerate AMGe methods (cf., [1, 2]).

\section{Introduction}

The purpose of this paper is to present some preliminary results on the performance of recently proposed overlapping Schwarz methods [3] for elliptic equations with high-contrast coefficients. These methods converge independent of the contrast and use a spectral construction of the coarse space. In this paper, we extend the methods and results of [3] to the multilevel case. The resulting multilevel methods are optimal in terms of the contrast. As it turns out, the resulting multilevel methods can be viewed as a version of previously proposed spectral agglomerate algebraic multigrid methods (or agglomerate $\varrho \mathrm{AMGe}$ ), originally in [1] (see also [6]) and its variant that allows

\footnotetext{
$\ddagger$ The work of Y.E. is partially supported by NSF and DOE.

$\S$ The work of this author was performed under the auspices of the U.S. DOE by Lawrence Livermore National Laboratory under Contract DE-AC52-07NA27344.
} 
for multilevel recursion without visiting the fine level in [2]. A computational survey of various AMGe methods is found in [5], see also [7].

The approach proposed in [3] for the two-level case, which we extend in the present contribution to the multilevel case, needs only identify the "vertices" of the agglomerates; no additional topological relations are required (assuming that we have somehow come up with an agglomeration algorithm or when geometric meshes are simply used as in our present experiments). The methods introduced here simplify the previously proposed similar spectral agglomerate AMGe methods in $[1,2]$. The simplification occurs due to the fact that the present method uses overlapping subdomains (unions of fine grid agglomerates (elements) that share a common vertex) as domains where the local eigenproblems are posed. To define the resulting coarse basis, a partition of unity is applied at the end (as commonly used). To implement the method, we need only an agglomeration algorithm and an algorithm to generate the vertices of the resulting agglomerates. No other topological relations need to be constructed or reduced Schur complements of local matrices need to be computed (as in [2] or [5]) and still the method allows for recursion without visiting the finest grid; see Section 3 below for details.

We apply the method to difficult elliptic finite element problems with discontinuous high-contrast coefficients (discontinuity, generally not aligned with the coarse elements). The two-level version of the method was proven in [3] to be robust with respect to the contrast. Our experiments that we present in this contribution show that the same result holds for a more practical multilevel extension of the method that we describe in the Section 3. The actual multiplicative and additive versions of the multilevel method are summarized after that in the following two sections, Sections 4-5. Finally, numerical results are presented in Section 7 .

\section{Notation and building tools}

Let $D \subset \mathbb{R}^{2}$ (or $\mathbb{R}^{3}$ ) be a polygonal domain. We would like to find $u^{*} \in H_{0}^{1}(D)$ such that

$$
a\left(u^{*}, v\right)=f(v) \quad \text { for all } v \in H_{0}^{1}(D),
$$

where the bilinear form $a$ and the functional $f$ are defined by

$$
a(u, v)=\int_{D} \kappa(x) \nabla u(x) \cdot \nabla v(x) d x
$$

and $f(v)=\int_{D} f(x) v(x) d x$ for all $u, v \in H_{0}^{1}(D)$. We allow discontinuous and high-contrast coefficient $\kappa$.

Let $\mathcal{T}_{h}=\mathcal{T}^{(0)}$ be a fine triangulation with mesh parameter $h=h^{(0)}$ and $V_{h}=V^{(0)}$ be the finite element space of piecewise linear functions on $\mathcal{T}^{(0)}$. The Galerkin formulation of (1) is to find $u^{*} \in V^{(0)}$ with $a\left(u^{*}, v\right)=f(v)$ for all $v \in V^{(0)}$, or in matrix form 


$$
A u^{*}=b,
$$

where for all $u, v \in V^{h}(D)$ we have $u^{T} A v=\int_{D} \kappa \nabla u \cdot \nabla v$, and $v^{T} b=\int_{D} f v$. We denote $A^{(0)}=A$. For each element $\tau \in \mathcal{T}^{(0)}$, let $A_{\tau}^{(0)}$ be the local finite element matrix. The fine-grid stiffness matrix $A^{(0)}$ can be obtained by the standard assembling procedure based on the local (element) matrices $A_{\tau}^{(0)}$, $\tau \in \mathcal{T}^{(0)}$.

It is sufficient to consider the case of piecewise constant coefficient $\kappa$. From now on we assume that $\kappa$ is a piecewise constant coefficient in $\mathcal{T}^{h}$ with value $\kappa=\kappa_{e}$ on each fine triangulation element $e \in \mathcal{T}^{h}$.

Introduce a coarser mesh $\mathcal{T}^{(1)} \supset \mathcal{T}^{(0)}$ with parameter $h^{(1)}$. We assume that each coarse element $T_{c} \in \mathcal{T}^{(1)}$ is the union of fine elements $\tau$ with $\tau \in \mathcal{T}^{(0)}$. Define also the subdomains $\{T\}$ as coarse vertex neighborhoods. For each subdomain $T$, there is coarse vertex $x_{T}$ such that $T=\cup\left\{T_{c}: x_{T} \in T_{c}\right\}$. Each subdomain $T$ contains only one coarse vertex $x_{T}$. Now, we define the subdomain matrices $A_{T}^{(0)}$. For interior floating subdomains, let $A_{T}^{(0)}$ be finite element Neumann matrix corresponding to that subdomain. If $T$ is a boundary subdomain let $A_{T}^{(0)}$ be the finite element matrix with homogeneous Neumann boundary conditions in the inner boundary $\partial T \cap D$ and homogeneous Dirichlet boundary conditions in the exterior boundary $\partial T \cap \partial D$. For any subdomain $T$, the matrix $A_{T}^{(0)}$ can be obtained by local assembling of finite element matrices as follows

$$
A_{T}^{(0)}=\sum_{\tau \in T} I_{\tau}^{(0)} A_{\tau}^{(0)} I_{\tau}^{(0) T},
$$

where $I_{\tau}^{(0)}$ is the extension by zero operator. Let $M_{T}^{(0)}$ the weighted mass matrix with coefficient $\kappa$ and of the same size of $A_{T}^{(0)}$. We solve the highcontrast eigenvalue problem

$$
A_{T}^{(0)} \phi_{k}=\lambda_{k} M_{T}^{(0)} \phi_{k}, \quad \phi_{k}=\phi_{k, T}, \quad \lambda_{1} \leq \lambda_{2} \ldots \lambda_{k} \leq \lambda_{k+1} \leq \ldots
$$

In practice, the mass matrix can be replaced with the diagonal of the respective stiffness matrix. This eigenvalue problem reveals the "small" part of the spectrum of the local subdomain matrix $A_{T}^{(0)}$. It can be shown that only a few small eigenvalues depend on the contrast, i.e., that they vanish asymptotically as the contrast increases. In particular, the number of these eigenvalues is the same as the number of isolated high-conductivity inclusions when homogeneous Neumann boundary conditions in (4) are considered. The idea is to include the corresponding eigenvector information into the coarse space. Let $\left\{D_{T}\right\}$ be a partition unity represented by nonnegative diagonal matrices $D_{T}$, that is, $\sum_{T} I_{T} D_{T} I_{T}^{T}=I d$, where $I d: V^{(0)} \rightarrow V^{(0)}$ is the identity operator and $I_{T}$ is the extension by zero operator. Let $L_{T}^{(0)}$ be an integer and define the coarse basis functions associated to the vertex $x_{T}$ by

$$
\Phi_{k}^{(1)}=D_{T} P^{(0)} \phi_{k}^{(0)}, \quad k=1, \ldots, L_{T}, \quad \Phi_{k}^{(1)}=\Phi_{k, T}^{(1)},
$$


where for convenience we define $P^{(0)}$ as the identity operator. These are the coarse degrees of freedom. That is, we define the coarse space

$$
V^{(1)}=\operatorname{Span}\left\{\Phi_{k}^{(1)}=\Phi_{k, T}^{(1)} T \text { subdomain, } 1 \leq k \leq L_{T}^{(0)}\right\} .
$$

We mention that one can modify the mass matrix $M_{T}^{(0)}$ to get coarse spaces with a smaller dimension, see [4].

We denote by $N_{T_{c}}^{(1)}$ the number of coarse degrees of freedom in a coarse element, or coarse basis functions with support containing a coarse element, $T_{c} \in \mathcal{T}^{(1)}$. With these (new) coarse basis functions we construct local matrices $A_{T_{c}}^{(1)}$.

Denote by $P^{(1)}$ the matrix whose columns are the coarse basis functions just defined including all subdomains $T$, that is

$$
P^{(1)}=\left[\Phi_{T, k}^{(1)}\right]_{T, 1 \leq k \leq L_{T}^{(0)}} .
$$

The matrix $P^{(1)}: V^{(1)} \rightarrow V^{(0)}$ is the interpolation from the coarse space $V^{(1)}$. We use the Galerkin relation to define the coarse-level "1" matrix

$$
A^{(1)}=P^{(1) T} A^{(0)} P^{(1)} .
$$

We now consider additional nested coarse meshes $\mathcal{T}^{(2)} \supset \cdots \supset \mathcal{T}^{(L)}$ with parameters $h^{(2)}, \ldots, h^{(\mathcal{L})}$ respectively. The procedure described above can be called recursively to construct coarse spaces $V^{(\ell)}$ and projections $P^{(\ell)}: V^{(\ell)} \rightarrow$ $V^{(0)}$. At level $\ell$, we consider the coarser triangulation $\mathcal{T}^{(\ell+1)}$ and we construct as before: local element matrices, $A_{\tau}^{(\ell)}, \tau \in \mathcal{T}^{(\ell)}$; subdomain local matrices, $A_{T}^{(\ell)}, M_{T}^{(\ell)}, T$ subdomain; coarse basis functions,

$$
\Phi_{k}^{(\ell+1)}=D_{T} P^{(\ell)} \phi_{k}^{(\ell)}, \quad k=1, \ldots, L_{T}^{(\ell)} ;
$$

coarse projection, $P^{(\ell+1)}=\left[\Phi_{T, k}^{(\ell)}\right]_{T, k}$; and global $\ell+1$ level matrix defined by $A^{(\ell+1)}=P^{(\ell+1) T} A^{(0)} P^{(\ell+1)}$.

Observe that $P^{(\ell+1)}: V^{(\ell+1)} \rightarrow V^{(0)}$ where we have defined the coarser space

$$
V^{(\ell+1)}=\operatorname{Span}\left\{\Phi_{k}^{(\ell)}=\Phi_{k, T}^{(\ell+1)} T \text { subdomain, } 1 \leq k \leq L_{T}^{(0)}\right\} .
$$

Each eigenvalue problem is defined at a current level: $A_{T}^{(\ell)} \phi_{k}=\lambda_{k} M_{T}^{(\ell)} \phi_{k}$. These are small size eigenvalue problems. Observe that, in order to construct new coarse basis functions we interpolate the solution of the local weighted eigenvalue problems into the finest space $V^{(0)}$ and then apply the partition of unity. In order to obtain a genuine multilevel construction only a minor modification needs to be done. Definition (6) of the coarse basis functions changes to 


$$
\widetilde{\Phi}_{k}^{(\ell+1)}=\widetilde{D}_{T}^{(\ell)} \phi_{k}^{(\ell)}, \quad k=1, \ldots, L_{T}^{(\ell)} .
$$

Where $\widetilde{D}_{T}^{(\ell)}$ is now a partition of unity of the $\ell$ th (coarse) degrees of freedom. In this case the corresponding spaces $\widetilde{V}^{(\ell)}$ are nested, $\widetilde{V}^{(0)} \supset \widetilde{V}^{(1)} \supset \cdots \supset$ $\widetilde{V}^{(\mathcal{L})}$, with the corresponding interpolation $\widetilde{P}^{(\ell)}: \widetilde{V}^{(\ell+1)} \rightarrow \widetilde{V}^{(\ell)}$ and matrices $\widetilde{A}^{(\ell+1)}=\widetilde{P}^{(\ell+1) T} \widetilde{A}^{(\ell)} \widetilde{P}^{(\ell+1)}$. Usual recursively defined multigrid (or MG) (Vcycle) algorithms can be used based on this construction. The MG algorithm based on the non-nested spaces is summarized in the next section. It falls into the category of "subspace correction" methods.

\section{Multigrid method}

Now we describe the multigrid method. We recall that in Section 3 we constructed coarse (non-nested) subspaces $V^{(1)}, \ldots, V^{(\mathcal{L})}$ associated to the coarse triangulations $\mathcal{T}^{(1)} \subset \mathcal{T}^{(2)} \ldots \subset \mathcal{T}^{(\mathcal{L})}$. Given $x, b \in V^{(0)}$, we define $y=M G(x, b)$ as corresponding multigrid (V-cycle) operator with (multiplicative) Schwarz smoother with initial guess $x$ and right hand side $b$. A detailed description of the computations is presented in Figure 4. The operator $r \rightarrow M G(0, r), r \in V^{(0)}$, can be used as a preconditioner. We also consider the genuine multilevel (V-cycle) multigrid method with in the construction using the coarse basis functions defined in (7).

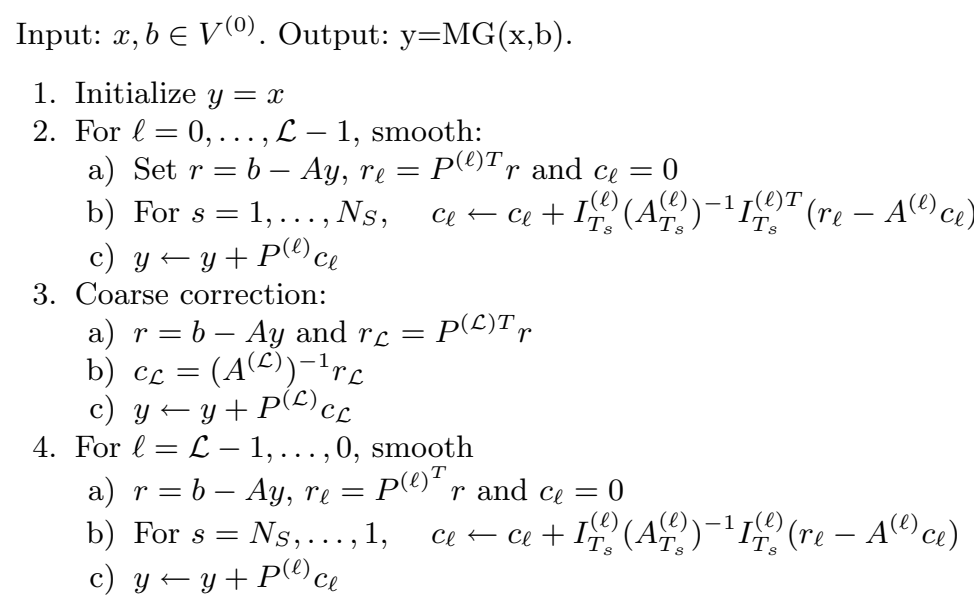

Fig. 1. Multigrid operator.

\section{Multilevel additive preconditioner (BPX)}

Now we define a BPX-like additive multilevel method with overlapping Schwarz method as smoother. Given $r \in V^{(0)}$ we define 


$$
M_{\text {add }}^{-1} r=\sum_{\ell=1}^{\mathcal{L}} \sum_{T} P^{(\ell)} I_{T}^{(\ell) T}\left(A_{T}^{(\ell)}\right)^{-1} I_{T}^{(\ell)} P^{(\ell) T} r
$$

where the second sum runs over all subdomains at level $\ell, \ell=1, \ldots, \mathcal{L}$. See [3] for a two-level version of this method. In [3], it is proved that $\operatorname{Cond}\left(M_{\text {add }}^{-1} A^{(0)}\right) \leq C\left(1+\frac{1}{h^{(1)} \lambda_{L+1}}\right)$, where $C$ is a constant independent of the contrast and $\lambda_{L+1}=\min _{T} \lambda_{L_{T}^{(1)}+1}$. If, in each subdomain, the right number of basis functions is chosen, then, the previous estimate becomes independent of the contrast. We note that the multilevel extension of [3] would require the solution of fine triangulation eigenvalue problems in each subdomain at every level. In our construction in Section 3, we solve eigenvalue problems at the actual level.

\section{Condition number bounds}

Now we present a simplified version of the condition number bounds for the methods described above. We recall that in this paper we are interested in the performance of the methods in terms of the contrast. We have the following results which proof uses tools developed in [1,2] and [3], see also [4]. A complete analysis of the proposed algorithms and more detailed numerical experiments are in progress.

Theorem 1. We have the condition number bounds for the preconditioned operators: $\operatorname{Cond}\left(M G(0, \cdot) A^{(0)}\right) \leq C$ and $\operatorname{Cond}\left(M_{\text {add }}^{-1} A^{(0)}\right) \leq D$, where the constants $C$ and $D$ depend on the number of levels and on the contrastindependent eigenvalues $\lambda_{k}=\lambda_{k, T}^{\ell}, k \geq L_{T}, T$ is a subdomain, $1 \leq \ell \leq \mathcal{L}$.

We stress upon the fact that our experiments indicate that the constants $C$ and $D$ above seem to be mesh-independent (at least in the case of geometric agglomerates). The independence (or exact dependence) of these estimates on the number of levels, as well as the analysis of the genuine multilevel algorithm, are subject of ongoing research.

\section{Numerical experiments}

In this section we present representative numerical experiments that show that the proposed methods have an optimal convergence in terms of contrast. We consider $D=[0,1] \times[0,1]$ and solve problem (1) with two different distributions of high-contrast coefficients $\kappa$. In the first experiment, we consider the coefficients depicted on the left of Figure 2. We use Preconditioned Conjugate Gradient (PCG) with the preconditioners described above, the multigrid, the genuine multilevel and the multilevel additive methods, see Sections 4 and 5 . The mesh and degrees of freedom information for the basis functions constructions in Section 3 is displayed in Table 1. The degrees of freedom information 
corresponding to the genuine multilevel method is similar and it is omitted. In Table 2 we present the (estimated) condition number of the preconditioned operator for different values of the contrast $\eta$. We iterate until the initial residual is reduced by a factor of $t o l=10^{-10}$. In our experiments, the selection of the number of small eigenvalues in each subdomain follows the criteria $L_{T}^{(\ell)}=\min _{k} \lambda_{k}>\rho$; for $\ell=0, \ldots, \mathcal{L}$, where $\rho$ is chosen based on the first large (order one) jump of the eigenvalues (c.f., $[3,2,5])$.
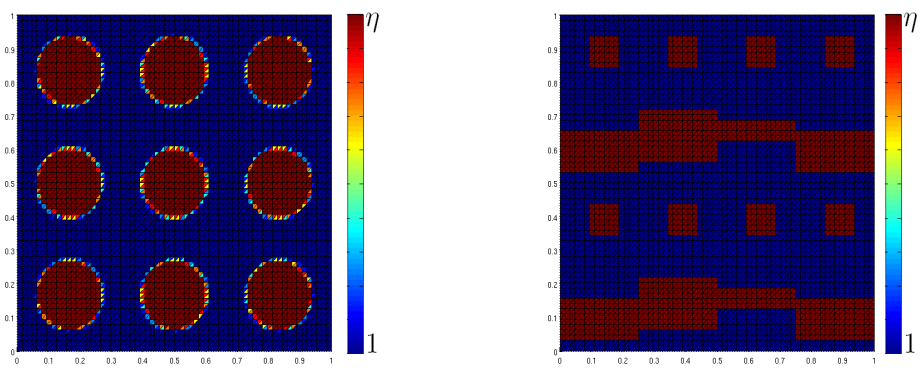

Fig. 2. Left: Coefficient corresponding to nine high-contrast inclusions. Red indicates the high-contrast part, $\kappa(x)=\eta$. Blue indicates value $\kappa(x)=1$. The results are displayed in Table 2 and the degrees of freedom information in Table 1. Right: Coefficient with inclusions and long channels. See Table 1 and 3 for mesh information and condition number results.

\begin{tabular}{|c|c|c|c|c|c|}
\hline Level & $\mathrm{h}$ & Subdomains & nodes (int) & dof MG-Ex. 1 & dof- MG Ex. 2 \\
\hline 0 & $1 / 64$ & $32 \times 32$ & $4225(3969)$ & $4225(3969)$ & $4225(3969)$ \\
1 & $1 / 32$ & $16 \times 16$ & $1089(961)$ & $1089(961)$ & $1089(961)$ \\
2 & $1 / 16$ & $8 \times 8$ & $289(225)$ & $289(225)$ & $289(225)$ \\
3 & $1 / 8$ & $4 \times 4$ & $81(49)$ & $117(77)$ & $113(78)$ \\
4 & $1 / 4$ & $2 \times 2$ & $25(9)$ & $77(45)$ & $38(19)$ \\
5 & $1 / 2$ & $1 \times 1$ & $9(1)$ & $45(9)$ & $27(6)$ \\
\hline \hline
\end{tabular}

Table 1. Mesh information and interior degrees of freedoms in each level for the coefficients depicted in Figure 2 ( $5^{\text {th }}$ and $6^{\text {th }}$ column, respectively). See Section 3.

In a second experiment we consider the coefficient depicted on the right of Figure 2. These coefficients have several inclusions and two long channels. The mesh information is contained in Table 1 and the condition number estimates are given Table 3 . As we observe from these numerical results that the proposed methods have optimal convergence in terms of the contrast. 


\begin{tabular}{|l|c|c|c|}
\hline$\eta$ & Multigrid & Genuine ML & Additive \\
\hline $10^{3}$ & 2.1389 & 2.1087 & 31.9844 \\
$10^{4}$ & 2.3288 & 2.3201 & 36.8847 \\
$10^{5}$ & 2.3612 & 2.4559 & 37.7580 \\
$10^{6}$ & 2.3647 & 2.4961 & 37.8532 \\
\hline
\end{tabular}

Table 2. Condition number estimates for the preconditioned CG. The information on the degrees of freedom is in Table 1 ( $5^{\text {th }}$ column).

\begin{tabular}{|l|c|c|c|}
\hline$\eta$ & Multigrid & Genuine ML & Additive \\
\hline $10^{3}$ & 1.7780 & 2.0010 & 27.0319 \\
$10^{4}$ & 1.7834 & 2.0751 & 27.4616 \\
$10^{5}$ & 1.7822 & 2.0837 & 27.5052 \\
$10^{6}$ & 1.7829 & 2.0846 & 27.5096 \\
\hline \hline
\end{tabular}

Table 3. Condition number estimates for the preconditioned CG. The information on the degrees of freedom is in Table 1 ( $6^{\text {th }}$ column).

\section{References}

[1] T. Chartier, R. Falgout, V.E. Henson, J. Jones, T. Manteuffel, S. McCormick, J. Ruge, and P.S. Vassilevski, Spectral AMGe, SIAM J. Scientific Computing 25 (2003), 1-26

[2] T. Chartier, R. Falgout, V.E. Henson, J. Jones, T. Manteuffel, S. McCormick, J. Ruge, and P.S. Vassilevski, Spectral element agglomerate $A M G e$, in Domain Decomposition Methods in Science and Engineering XVI, Lecture Notes in Computational Science and Engineering, SpringerVerlag, Berlin Heidelberg 55(2007), pp.'515-524.

[3] Y. Efendiev and J. Galvis, Domain decomposition preconditioners for multiscale flows in high contrast media, submitted.

[4] Y. Efendiev and J. Galvis, A domain decomposition preconditioner for multiscale high-contrast problems, presented in the Proceedings of DD19.

[5] I. Lashuk and P. S. Vassilevski, On some versions of the element agglomeration AMGe methods, Numerical Linear Algebra with Applications 15(2008), pp. 595-620.

[6] M. Brezina, C. Heberton, J. Mandel and P. Vanek, An iterative method with convergence rate chosen a priori, UCD/CCM Report 140, 1999 (available at http://www-math.cudenver.edu/jjmandel/papers).

[7] P. S. Vassilevski. Multilevel block-factorization preconditioners. Matrixbased analysis and algorithms for solving finite element equations, Springer, New York, 2008. 